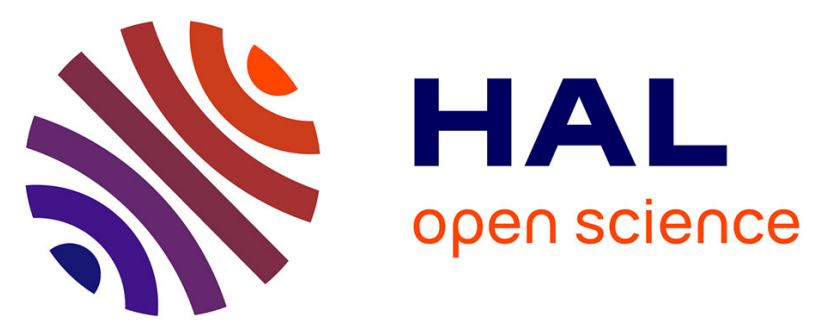

\title{
AHNAK C-Terminal Peptide Membrane Binding-Interactions between the Residues 5654-5673 of AHNAK and Phospholipid Monolayers and Bilayers
} Xiaolin Yan, Francis Noël, Isabelle Marcotte, Christine Dewolf, Dror E.

Warschawski, Elodie Boisselier

\section{To cite this version:}

Xiaolin Yan, Francis Noël, Isabelle Marcotte, Christine Dewolf, Dror E. Warschawski, et al.. AHNAK C-Terminal Peptide Membrane Binding-Interactions between the Residues 5654-5673 of AHNAK and Phospholipid Monolayers and Bilayers. Langmuir, 2019, 36 (1), pp.362-369. 10.1021/acs.langmuir.9b02973 . hal-02567660

\section{HAL Id: hal-02567660 https://hal.science/hal-02567660}

Submitted on 30 Nov 2020

HAL is a multi-disciplinary open access archive for the deposit and dissemination of scientific research documents, whether they are published or not. The documents may come from teaching and research institutions in France or abroad, or from public or private research centers.
L'archive ouverte pluridisciplinaire HAL, est destinée au dépôt et à la diffusion de documents scientifiques de niveau recherche, publiés ou non, émanant des établissements d'enseignement et de recherche français ou étrangers, des laboratoires publics ou privés. 


\title{
AHNAK C-terminal peptide membrane binding -
}

\section{Interactions between the residues $5654-5673$ of}

\section{AHNAK and phospholipid monolayers and bilayers}

\author{
Xiaolin Yan, ${ }^{1,2}$ Francis Noël, ${ }^{1,2}$ Isabelle Marcotte, ${ }^{3}$ Christine E. DeWolf, ${ }^{4}$ Dror E. \\ Warschawski, ${ }^{3,5}$ and Elodie Boisselier ${ }^{* 1,2}$
}

\begin{abstract}
${ }^{1}$ Department of ophthalmology, Faculty of medicine, Université Laval, Quebec City, Canada; ${ }^{2}$ CUO-Recherche, Centre de recherche du CHU de Québec, Hôpital du Saint-Sacrement, CHU de Québec, Quebec City, Canada; ${ }^{3}$ Departement of chemistry, Faculty of sciences, Université du Québec à Montréal, Montreal, Canada; ${ }^{4}$ Department of Chemistry and Biochemistry and Centre for NanoScience Research, Concordia University; 5 UMR 7099, CNRS-Université Paris Diderot, Institut de Biologie Physico-Chimique, Paris, France
\end{abstract}

KEYWORDS: AHNAK, Membrane binding, Langmuir monolayer, Ellipsometry, PROCSA.

\begin{abstract}
The dysferlin membrane repair complex contains a small complex, S100A10annexin A2, which initiates membrane repair by recruiting the protein AHNAK to the membrane, where it interacts via binding sites in the C-terminal region. However, no molecular data are available for the membrane binding of the various proteins involved in this complex. Therefore, the present study investigated the membrane binding of AHNAK to elucidate its role in the cell membrane repair process. A chemically synthesized peptide (pAHNAK), comprising the 20 amino
\end{abstract}


acids in the C-terminal domain of AHNAK, was applied to Langmuir monolayer models, and the binding parameters and insertion angles were measured with surface tensiometry and ellipsometry. The interaction of pAHNAK with lipid bilayers was studied using ${ }^{31} \mathrm{P}$ solid-state nuclear magnetic resonance. pAHNAK preferentially and strongly interacted with phospholipids that comprised negatively charged polar head groups with unsaturated lipids. This finding provides a better understanding of AHNAK membrane behavior and the parameters that influence its function in membrane repair.

\section{INTRODUCTION}

AHNAK, also known as desmoyokin, ${ }^{1}$ is an extremely large protein owing to its molecular weight of $629.101 \mathrm{kDa}$ (according to its sequence in the UniProt Knowledge Base, Q09666). AHNAK comprises three domains: the N-terminal, C-terminal, and central domains, the latter containing approximately 4300 amino acids with 128 -residue repetitive elements. ${ }^{1-2}$ Although there are various protein interaction sites throughout the protein, the majority are located within the C-terminal domain. ${ }^{1}$ AHNAK has been shown to play central roles in three important processes: cell signaling and cell contacts, ${ }^{1,3-5}$ calcium channel regulation, ${ }^{1,6-7}$ and membrane repair. ${ }^{1,8-9}$ AHNAK is found in the dysferlin membrane repair complex, which also includes a heterotetrametric S100A10-annexin A2 complex. ${ }^{10-13}$ In the presence of calcium, the dysferlin membrane repair complex promotes the fusion of exocytosis vesicles, which expand and reseal the inner side of the cell membrane. ${ }^{14-17}$ Moreover, AHNAK acts as a marker of enlargeosomes, which are vesicles located in the cytoplasmic rim beneath the cell membrane. ${ }^{1,9,18-19}$ Following a calcium flux signal, the enlargeosomes undergo exocytosis and play a crucial role in membrane repair via a process in which the S100A10-annexin A2 complex, to which AHNAK shows a high affinity, recruits AHNAK to the membrane, where it forms a platform through the binding sites in its C- 
terminal domain $\left(\mathrm{G}_{5654}-\mathrm{L}_{5673}\right) .{ }^{13}$ Understanding the interaction between the lipid membrane and this membrane repair complex is important for a better understanding of the cell membrane repair process. However, there are no data available regarding the interaction between AHNAK and the lipids that comprise the membrane.

The present study investigated the membrane binding of AHNAK by applying various biophysical techniques. A chemically synthesized peptide (pAHNAK), which comprised the 20 amino acids $\left(\mathrm{G}_{5654}-\mathrm{L}_{5673}\right)$ of the $\mathrm{C}$-terminal domain of AHNAK, was used with two membrane models: the Langmuir monolayer model and a lipid bilayer model. These were studied using surface tensiometry, ellipsometry, and ${ }^{31} \mathrm{P}$ solid-state nuclear magnetic resonance (NMR) spectroscopy. The Langmuir monolayer model mimics an asymmetric cell membrane and facilitates control over several parameters, including surface pressure and buffer composition as well as the physical state of the associated lipids. ${ }^{20-21}$ Multilayer vesicles contain several lipid bilayers; they are relatively simple to prepare, require no support, and provide a sufficient signal for NMR analysis. ${ }^{22}$ The experiments with the Langmuir monolayer model demonstrated that pAHNAK has a high propensity to interact with monounsaturated lipids. The ellipsometry and ${ }^{31} \mathrm{P}$ solid-state NMR studies suggested that pAHNAK inserted more deeply into a monolayer of the monounsaturated lipid DOPS (1,2-dioleoyl-sn-glycero-3-phospho-L-serine) than with monolayers of two other monounsaturated lipids, DOPE (1,2-dioleoyl-sn-glycero-3-phosphoethanolamine) and DOPC (1,2-dioleoyl-sn-glycero-3-phosphocholine). These findings provide a better understanding of the membrane behavior of AHNAK during membrane repair as well as its other roles involving lipids.

\section{EXPERIMENTAL SECTION}


Materials. The peptide pAHNAK, which comprises the 20 amino acids of the C-terminal domain of AHNAK ( $\mathrm{G}_{5654}$ K V T F P K M K I P K F T F S G R E L 5673$)$, was chemically synthesized with the standard Fmoc solid-phase synthesis method, in the medicinal chemistry platform of the CHU de Québec (Quebec, Canada), with a purity exceeding 95\%. The pI was calculated with an online peptide property calculator from Genscript (https://www.genscript.com/tools/peptide-property-calculator).

The deionized water used for preparing all the buffer solutions was obtained from a Barnstead Nanopure system (Barnstead, Dubuque, IA, USA); its resistivity and surface tension at $20^{\circ} \mathrm{C}$ were 18.2 $\mathrm{M} \Omega \cdot \mathrm{cm}$ and $72 \mathrm{mN} / \mathrm{m}$, respectively. Tris Base and butylated hydroxytoluene were purchased from Fisher Scientific (Hampton, NH, USA), and hydrochloric acid from VWR International (Radnor, PA, USA). High performance liquid chromatography-grade chloroform and methanol were obtained from Laboratoire Mat (Quebec, Canada). The following phospholipids were obtained from MilliporeSigma (Burlington, MA, USA): 1,2-dipalmitoyl-sn-glycero-3phosphoethanolamine (DPPE), 1,2-dipalmitoyl-sn-glycero-3-phospho-L-serine (sodium salt) (DPPS), 1,2-dipalmitoyl-sn-glycero-3-phosphocholine (DPPC), 1,2-distearoyl-sn-glycero-3phosphoethanolamine (DSPE), 1,2-distearoyl-sn-glycero-3-phospho-L-serine (sodium salt) (DSPS), 1,2-distearoyl-sn-glycero-3-phosphocholine (DSPC), 1,2-dioleoyl-sn-glycero-3phosphoethanolamine (DOPE), 1,2-dioleoyl-sn-glycero-3-phospho-L-serine (sodium salt) (DOPS), 1,2-dioleoyl-sn-glycero-3-phosphocholine (DOPC), 1,2-didocosahexaenoyl-sn-glycero3-phosphoethanolamine (DDPE), 1,2-didocosahexaenoyl-sn-glycero-3-phospho-L-serine (sodium salt) (DDPS), and 1,2-didocosahexaenoyl-sn-glycero-3-phosphocholine (DDPC). All the lipid solutions were prepared in chloroform, with the exception of DSPS, which was prepared in chloroform, methanol, and water in the proportions $65: 25: 4(\mathrm{v} / \mathrm{v})$, at a concentration of 0.1 to 
$0.2 \mathrm{mg} / \mathrm{mL}$. Unsaturated lipids were stored under argon in the presence of the antioxidant butylated hydroxytoluene $(5 \mu \mathrm{g} / \mathrm{mL}),{ }^{23}$ and saturated lipids were stored under atmospheric air. All the lipids and lipid solutions were stored at $-20^{\circ} \mathrm{C}$.

Surface Pressure Measurements. The protein binding parameters were determined by the Wilhelmy method to determine the surface tension. ${ }^{24}$ The surface pressure (П) was measured with a DeltaPi4 microtensiometer (Kibron Inc., Helsinki, Finland), using a $1000-\mu \mathrm{L}$ Teflon ${ }^{\circledR}$ trough (diameter: $18 \mathrm{~mm}$, depth: $5 \mathrm{~mm}$ ). A Plexiglass box was used to control the humidity for the experiments, and the experimental temperature was maintained at $20^{\circ} \mathrm{C} \pm 1{ }^{\circ} \mathrm{C}$. The trough subphase comprised a $1000-\mu \mathrm{L}$ buffer of $20 \mathrm{mM}$ Tris at $\mathrm{pH}$ 8.0. The saturating concentration was determined by injecting increasing volumes of pAHNAK underneath the surface of the subphase; it was found to be $13.7 \mu \mathrm{g} / \mathrm{mL}$ for a surface pressure of $10.5 \mathrm{mN} / \mathrm{m}$. This concentration corresponded to the onset of monolayer saturation and was used for all the following experiments.

In the subsequent experiments, a few microliters of the phospholipid solution for testing were spread onto the subphase to obtain the desired initial surface pressure $\left(\Pi_{\mathrm{i}}\right)$. This was then left until the spreading solvent had completely evaporated and the film reached equilibrium; the time required for this depended on the type of lipid, the spreading volume, and the initial surface pressure. ${ }^{25-26}$ The pAHNAK was then injected underneath the lipid monolayer at the saturating concentration of $13.7 \mu \mathrm{g} / \mathrm{mL}$. The interaction between the pAHNAK and the phospholipid monolayer was monitored during the experiment with surface pressure kinetics until the equilibrium surface pressure $\left(\Pi_{\mathrm{e}}\right)$ was reached. The change in surface pressure variation $(\Delta \Pi)$ induced by the protein was calculated as $\Pi_{\mathrm{e}}-\Pi_{\mathrm{i}}$.

Determination of the Binding Parameters. The binding parameters were determined and the uncertainties calculated as previously described..$^{20,23,25-31}$ Briefly, the change in surface pressure 
$(\Delta \Pi)$ after the injection of protein was plotted as a function of $\Pi_{i}$ and fitted by linear regression. The maximum insertion pressure was then determined from the intersection of the plot with the $x$ axis and the synergy was calculated as $1+$ the slope. The uncertainty of the MIP was calculated from the covariance of the experimental data on the linear regression and the uncertainty of the synergy was calculated using $\left(\sigma\left(\Pi_{e}\right)\left(1-r^{2}\right)^{1 / 2}\right) /\left(\sigma\left(\Pi_{i}\right)(n-2)^{1 / 2}\right)$, where $\sigma$ is the standard deviation, $\mathrm{r}$ is the correlation coefficient, and $\mathrm{n}$ is the number of points. The calculations of the binding parameters and their uncertainties were made using an online software calculator (http://www.crchudequebec.ulaval.ca/BindingParametersCalculator).

Ellipsometry Measurements. The ellipsometry measurements were performed with a polarizer compensator specimen analyzer null imaging ellipsometer (I-Elli2000; Nanofilm, now Accurion $\mathrm{GmbH}$, Goettingen, Germany) using a $532 \mathrm{~nm}, 50 \mathrm{~mm} \mathrm{Nd:YAG} \mathrm{laser.} \mathrm{The} \mathrm{measurements} \mathrm{were}$ made at an angle of incidence of $50^{\circ}$ to the air-water interface (the Brewster angle of the air-water interface for pure water is $53.12^{\circ}$ ). The compensator was set at $20.00^{\circ}$ and the laser output was $100 \% .^{26,32}$ The ellipsometric angles $\Delta$ were measured for different regions of interest (size $\geq 20$ $\mu \mathrm{m})$ to reduce the influence of the lateral structure or defects within the beam spot and preserve spatial information, ensuring the results were accurate and reproducible. ${ }^{33}$ Each data point represented the mean of ten experimental measurements. A home-made Teflon ${ }^{\circledR} 5000-\mu \mathrm{L}$ trough was used in these experiments, with a subphase buffer containing $20 \mathrm{mM}$ Tris at $\mathrm{pH}$ 8.0. A tensiometer (Nima Technology, Coventry, UK) was used to measure the surface pressure by the Wilhelmy method. All the experiments were performed at $20^{\circ} \mathrm{C} \pm 1^{\circ} \mathrm{C}$. The protein concentration in the subphase was $13.7 \mu \mathrm{g} / \mathrm{mL}$.

Ellipsometric Angle Determination. The ellipsometric angle of the subphase (i.e., the buffer), $\Delta_{\text {subphase, }}$ was measured as the baseline at the beginning of each experiment. Before adding a lipid 
monolayer, pAHNAK was injected at the saturating concentration of $13.7 \mu \mathrm{g} / \mathrm{mL}$ and the surface pressure kinetics were monitored until the surface pressure reached equilibrium, measuring the ellipsometric angles every $10 \mathrm{~min}$. For the lipid baseline measurements, a few microliters of phospholipid solution was spread on the subphase to obtain an initial pressure of $10 \mathrm{mN} / \mathrm{m}$. Ellipsometric angles were measured for pAHNAK, DOPC, DOPE, and DOPS $\left(\Delta_{\mathrm{pAHNAK}}, \Delta_{\mathrm{DOPC}}\right.$, $\Delta_{\text {DOPE }}$, and $\Delta_{\text {DOPS }}$, respectively), correcting each by subtracting the value of $\Delta_{\text {subphase }}$ to avoid the influence of the subphase. For the measurements in the presence of both a lipid and the protein, the lipid was first spread to the desired surface pressure $(10 \mathrm{mN} / \mathrm{m})$ and then pAHNAK was injected at $13.7 \mu \mathrm{g} / \mathrm{mL}$. Ellipsometric angles were measured every 15 min until the surface pressure reached the equilibrium, at which point $\Delta_{\text {pAHNAK-lipid }}$ was determined. The experimental value $\Delta_{\text {pAHNAK-lipid }}$ was compared with the sum of $\Delta_{\text {pAHNAK }}$ and $\Delta_{\text {lipid }}$ as the expected value.

Multilayer vesicles preparation. The samples for NMR were prepared as follows. A mixture of $6.7 \mathrm{mg}$ of DOPE, DOPS, and DOPC in a 1:1:1 molar ratio in a chloroform solution was kept on ice and dried under nitrogen steam, followed by lyophilization overnight to remove the residual organic solvent. Then, the lipid mixture was hydrated by adding $2 \mathrm{mg}$ of pAHNAK solubilized in $26 \mu \mathrm{L}$ buffer ( $20 \mathrm{mM}$ Tris at $\mathrm{pH} 8.0$ ). Alternatively, $26 \mu \mathrm{L}$ buffer without protein was added to the lipid mixture as a control. The mixture was vortexed and freeze-thawed three times (for $10 \mathrm{~min}$ at $-20^{\circ} \mathrm{C}$ followed by $10 \mathrm{~min}$ at room temperature) to create multilamellar lipid vesicles. A disposable Kel-F insert was filled with about 8-20 mg of the sample and then placed inside a 4-mm rotor.

${ }^{31} \mathrm{P}$ Solid-State NMR Measurements. Each experiment was performed twice at $20^{\circ} \mathrm{C}$ to corroborate the membrane binding measurements and at $37^{\circ} \mathrm{C}$ to mimic the physiological temperature of the human body. 
PROCSA experiments were performed using a 400-MHz solid-state NMR Bruker Avance IIIHD wide-bore spectrometer (Bruker, Milton, Ontario, Canada), operating at a ${ }^{31} \mathrm{P}$ frequency of $162 \mathrm{MHz}$, with a 4-mm double resonance MAS probe. ${ }^{34}$ The samples were spun at $6 \mathrm{kHz}$, during which the temperature was calibrated and corrected, with an equilibration time of at least 15 min between the steps in temperature. The phosphorus $\left(90^{\circ}\right)$ pulse length was $3 \mu \mathrm{s}$; the PROCSA pulses were applied at a field strength of around $25 \mathrm{kHz}$. Proton decoupling during acquisition was performed with two-pulse phase modulation at a field strength of $25 \mathrm{kHz}$. Two-dimensional spectra were acquired with 256 scans for each of the 32 rows and a recycle delay of $3 \mathrm{~s}$, taking a total time of $7 \mathrm{~h}$. The processing was performed with automatic baseline correction and $5 \mathrm{~Hz}$ of line broadening, using the Bruker TopSpin 3.5 interface. The precision in the chemical-shift anisotropy determination was $\pm 2 \mathrm{ppm}$.

Measurement of infrared spectra. Infrared spectra were measured with a Golden Gate ATR accessory (Specac, Woodstock, NY) installed in a Thermo Nicolet 6700 Fourier transform infrared (FTIR) spectrometer. The multilamellar vesicles were centrifuged at $15000 \mathrm{~g}$ during 15 minutes before analysis. The spectrum of the multilamellar vesicles alone was subtracted from that of pAHNAK in the presence of the vesicles to get the spectrum of the peptide in the presence of the vesicles.

\section{RESULTS AND DISCUSSION}

Determination of the Membrane Binding Parameters of pAHNAK. It is well established that AHNAK is a marker of enlargeosomes. ${ }^{1,9,18-19}$ However, the lipid composition of enlargeosomes remains to be determined; it is only known that they probably contain cholesterol, phospholipids, sphingolipids, and lipid rafts. ${ }^{19}$ Phospholipids comprise a hydrophilic polar head group, which is 
negatively charged or zwitterionic, and two hydrophobic acyl chains, which can both be saturated, both unsaturated, or one saturated and one unsaturated. The different characteristics of the phospholipids influence their interactions with proteins. For example, lipids with a negatively charged head group (such as the polar head group of phospho-L-serine) are more likely to interact with positively charged proteins. Size can also have an influence: phosphocholine and phosphoethanolamine are both zwitterionic, but phosphocholine is significantly larger, which alters the polar head group and chain packing constraints and can influence lipid-protein interactions, especially for those where the protein is inserted into the membrane. ${ }^{35-36}$ The presence of unsaturated bonds in the acyl chains influences the physical state and phase of the lipid, which affects lipid-protein interactions. For these reasons, this study of pAHNAK membrane binding used 12 phospholipids that reflected these various properties. Six of those studied with the Langmuir monolayer model had saturated acyl chains: DPPE, DPPS, and DPPC are diC16:0 (i.e., each has two acyl chains of palmitic acid, which has 16 carbons with 0 unsaturation), and DSPE, DSPS, and DSPC are diC18:0 (with stearic acid chains). The other six phospholipids were unsaturated: DOPE, DOPS, and DOPC are diC18:1 (oleic acid), and DDPE, DDPS, and DDPC are diC22:6 (docosahexaenoic acid). One of the binding parameters measured in these experiments, the maximum insertion pressure (MIP), is the pressure at which a protein can no longer insert into a lipid membrane. ${ }^{27}$ Higher values of MIP indicate stronger affinity between the protein and the lipid membrane. The other binding parameter measured was the synergy, which represents the type of interaction observed between the protein and the lipid monolayer. When the synergy has a positive value, there is an attractive interaction between the protein and lipid; when the synergy is negative, this represents repulsion between them. The synergy and MIP values for pAHNAK with the 12 lipids tested are presented in Table 1 and Figure 1. 
Table 1. Synergy and maximum insertion pressure (MIP) values for pAHNAK with the lipids tested.

\begin{tabular}{|l|l|l|}
\hline Name & Synergy & MIP $(\mathrm{mN} / \mathrm{m})$ \\
\hline DPPE & $0.59 \pm 0.04$ & $21.9 \pm 2.3$ \\
\hline DPPS & $0.11 \pm 0.10$ & $22.3 \pm 2.2$ \\
\hline DPPC & $0.45 \pm 0.08$ & $19.5 \pm 2.5$ \\
\hline DSPE & $0.66 \pm 0.03$ & $17.7 \pm 1.5$ \\
\hline DSPS & $0.45 \pm 0.04$ & $24.8 \pm 1.5$ \\
\hline DSPC & $0.02 \pm 0.13$ & $19.0 \pm 3.0$ \\
\hline DOPE & $0.51 \pm 0.04$ & $38.8 \pm 3.8$ \\
\hline DOPS & $0.55 \pm 0.03$ & $58.0 \pm 5.6$ \\
\hline DOPC & $0.43 \pm 0.06$ & $29.7 \pm 3.4$ \\
\hline DDPE & $0.42 \pm 0.03$ & $32.3 \pm 1.7$ \\
\hline DDPS & $0.33 \pm 0.06$ & $28.4 \pm 3.2$ \\
\hline DDPC & $0.31 \pm 0.05$ & $22.0 \pm 2.0$ \\
\hline & & \\
\hline & & \\
\hline & & \\
\hline & & \\
\hline & & \\
\hline & & \\
\hline & & \\
\hline & & \\
\hline
\end{tabular}

Notes: The lipids are defined in the Experimental Section. Chains: DP, dipalmitoyl; DS, distearoyl; DO, dioleoyl; DD, didocosahexaenoyl. Polar head groups: PE, phosphoethanolamine; PS, phosphoserine; PC, phosphocholine. 
Almost all the synergy values were higher than zero (Figure 1a), indicating that pAHNAK interacted positively with all the lipids. However, the synergy value for DSPC was close to zero, suggesting neither attraction nor repulsion between pAHNAK and the DSPC phospholipid monolayer. This observation means than the packing of the DS acyl chains leads to a lipid organization which is less favorable to the binding of pAHNAK than that of other acyl chains with the same PC polar head group, despite similar MIP values (19.5 and $19.0 \mathrm{mN} / \mathrm{m}$ for DPPC and DSPC, respectively). The value for DPPS $(0.11 \pm 0.10)$ was also distinctly lower than that for the other lipids. With the saturated phospholipids, those with the phosphoethanolamine polar head group showed the highest synergy values, of $0.59 \pm 0.04$ for DPPE and $0.66 \pm 0.03$ for DSPE. With the unsaturated phospholipids, the synergy values were higher for the lipids with dioleoyl acyl chains than for those with the didocosahexaenoyl acyl chains, indicating that the single unit of saturation found in dioleoyl favors pAHNAK binding. The highest synergy observed for the unsaturated phospholipids was that between pAHNAK and DOPS, with a value of $0.55 \pm 0.03$.

The MIP values for all the saturated phospholipids were $<30 \mathrm{mN} / \mathrm{m}$ (Figure $1 \mathrm{~b}$ ), indicating that pAHNAK cannot insert into domains composed mainly of these types of phospholipids in a physiological context. The membrane lateral pressure has been estimated to be about $30 \mathrm{mN} / \mathrm{m},{ }^{26}$, ${ }^{37-42}$ so MIP values $<30 \mathrm{mN} / \mathrm{m}$ would suggest that pAHNAK insertion into a membrane would be unlikely. Moreover, all the MIP values are above the equilibrium surface pressure observed with the peptide alone $(10.5 \mathrm{mN} / \mathrm{m})$, highlighting the high attraction of the peptide to the lipid membrane in the presence of the different phospholipids. The MIP values were quite similar across all the saturated phospholipids, indicating that the interaction between pAHNAK and the phospholipid was not affected by the length of the distearoyl and dipalmitoyl acyl chains. pAHNAK clearly cannot penetrate the close-packed lipids in the condensed phase. However, MIP 
values were higher for the unsaturated phospholipids than for the saturated phospholipids, indicating that pAHNAK preferentially interacts with and inserts into unsaturated lipids. Furthermore, the MIP values were higher for monounsaturated phospholipids than for the polyunsaturated phospholipids with the same polar head group, which suggests that the thickness of the acyl chain region influences binding, in addition to the lipid phase, as previously reported. ${ }^{29}$ The highest MIP value was with the DOPS monolayer $(58 \pm 5.6 \mathrm{mN} / \mathrm{m})$; together with the high synergy value $(0.55 \pm 0.03)$, this suggests that pAHNAK is highly likely to interact with physiological domains that have a high proportion of DOPS. High MIP and synergy values were also found for DOPE and DOPC (MIP: $38.8 \pm 3.8$ and $29.7 \pm 3.4 \mathrm{mN} / \mathrm{m}$, respectively; synergy: $0.51 \pm 0.04$ and $0.43 \pm 0.06$ ). This suggests the following preferential interaction order for pAHNAK: DOPS $>$ DOPE $>$ DOPC. This observation is consistent with pAHNAK having a pI of 11.25 (calculated with an online peptide property calculator from Genscript), which results in the protein having a positive charge at $\mathrm{pH}$ 8.0. Indeed, the pAHNAK sequence is G K V T F P K M K I P K F T F S G R E L, with positively and negatively charged amino acids in red and blue, respectively. pAHNAK has thus $25 \%$ of its amino acids carrying a positive charge, suggesting that pAHNAK may interact with the DOPS monolayer through electrostatic interactions. Steric hindrance seems also to have an effect on pAHNAK binding, as the phosphocholine head group is larger than the phosphoethanolamine head group, suggesting that a smaller phospholipid head group promotes pAHNAK interaction. Indeed, despite the fact that DOPE, DOPC and DOPS contain the same acyl chains, the molecular area per lipid is different for the three polar head groups and are $85 \AA^{2}, 94 \AA^{2}$ and $97 \AA^{2}$ per lipid respectively for a surface pressure of $5 \mathrm{mN} / \mathrm{m} .{ }^{43-}$ 44 Thus phospholipid packing favors DOPS which offers more space for the interaction of the peptide. While the values of the binding parameters observed with DDPS are not higher than the 
ones observed with DDPE and DDPC, the same trend is observed for the influence of the polar head group with didocosahexaenoyl acyl chains. The single double bond of the dioleoyl acyl chains seems here to favor electrostatic interactions with negatively charged phosphoserine polar head groups, which may be attributed to differences in acyl chain region thickness.
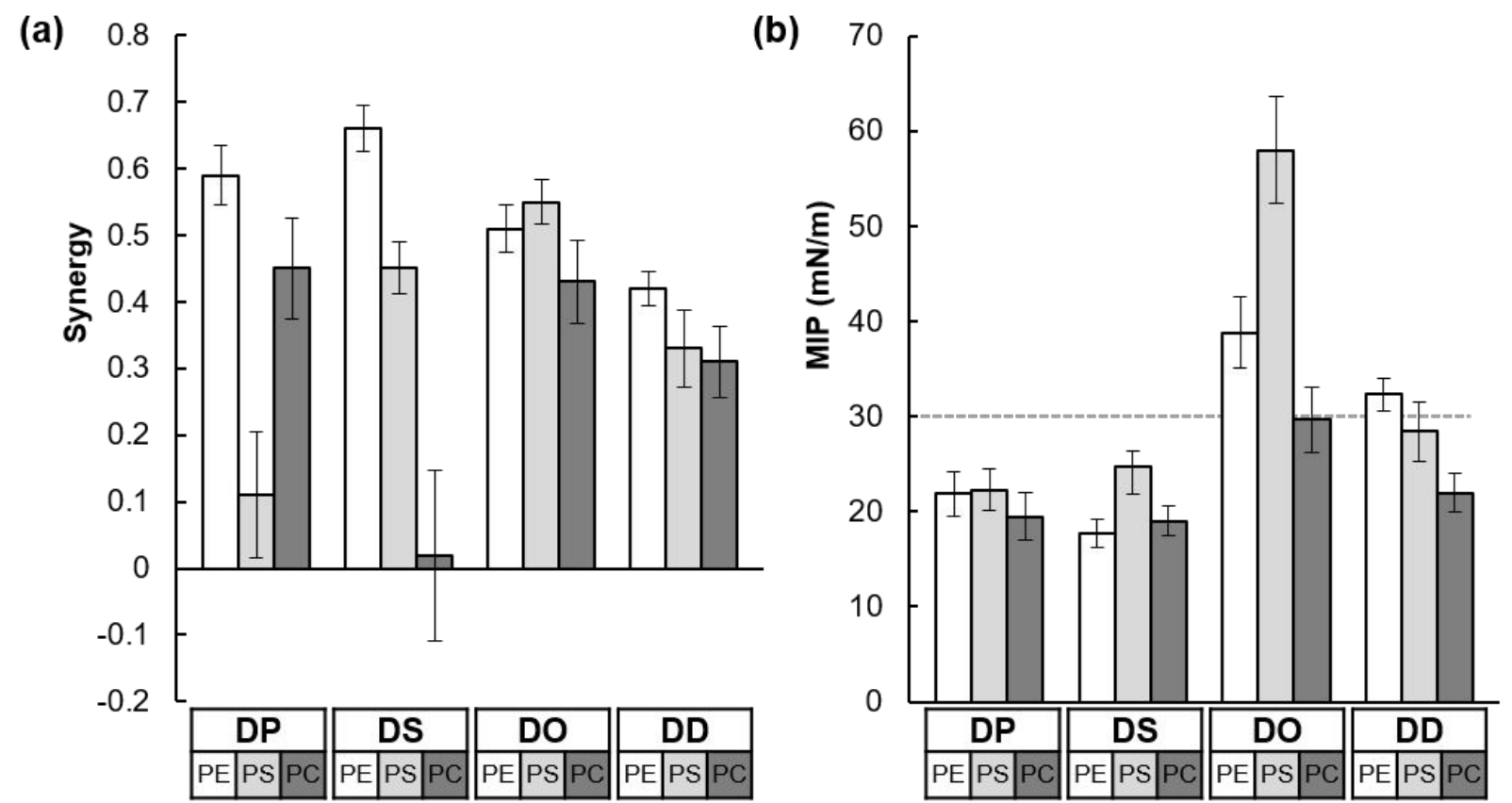

Figure 1. Synergy (a) and maximal insertion pressure (MIP) (b) of pAHNAK for the 12 lipids. Chains: DP, dipalmitoyl; DS, distearoyl; DO, dioleoyl; DD, didocosahexaenoyl. Polar head groups: PE, phosphoethanolamine; PS, phosphoserine; PC, phosphocholine.

Insertion Depth of pAHNAK into the Phospholipid Monolayers. To better understand the membrane binding between pAHNAK and the monounsaturated phospholipids, ellipsometry was used to compare the insertion depths of pAHNAK into DOPE, DOPS, and DOPC monolayers. The ellipsometric angle $\Delta$ of pAHNAK alone $\left(\Delta_{\mathrm{pAHNAK}}\right)$ was $0.87^{\circ} \pm 0.06$. For an initial surface 
pressure of $10 \mathrm{mN} / \mathrm{m}$, the ellipsometric angles for the DOPS $\left(\Delta_{\mathrm{DOPS}}\right), \mathrm{DOPE}\left(\Delta_{\mathrm{DOPE}}\right)$, and DOPC $\left(\Delta_{\text {DOPC }}\right)$ monolayers were $1.26^{\circ} \pm 0.19^{\circ}, 1.21^{\circ} \pm 0.25^{\circ}$, and $1.12^{\circ} \pm 0.24^{\circ}$, respectively. With the mixed lipid-protein systems, obtained after injecting the protein underneath the film and allowing the surface pressure to reach equilibrium, the ellipsometric angles represent the interaction between pAHNAK and the lipid monolayer. The values for $\Delta_{\mathrm{pAHNAK}-\mathrm{DOPS}}, \Delta_{\mathrm{pAHNAK}-\mathrm{DOPE}}$, and $\Delta_{\text {pAHNAK-DOPC }}$ were $1.79^{\circ} \pm 0.21^{\circ}, 2.08^{\circ} \pm 0.19^{\circ}$, and $2.32^{\circ} \pm 0.21^{\circ}$, respectively (Table 2 and Figure 2). In the presence of a lipid monolayer, the expected $\Delta$ value can be calculated as the sum of $\Delta_{\text {pAHNAK }}$ and $\Delta_{\text {DOPS }}$, as described in the Experimental Section. These expected values for DOPS, DOPE, and DOPC were $2.13^{\circ} \pm 0.25^{\circ}, 2.08^{\circ} \pm 0.31^{\circ}$, and $1.99^{\circ} \pm 0.30^{\circ}$, respectively.

Table 2. Comparison of expected and experimental values of the ellipsometric angles observed for pAHNAK interacting with monolayers of the monounsaturated phospholipids DOPS, DOPE, and DOPC at $20^{\circ} \mathrm{C}$.

\begin{tabular}{|l|l|l|}
\hline Interaction & Experimental value $\left(^{\circ}\right)$ & Expected value $\left(^{\circ}\right)$ \\
\hline pAHNAK-DOPS & $1.79 \pm 0.21$ & $2.13 \pm 0.25$ \\
\hline pAHNAK-DOPE & $2.08 \pm 0.19$ & $2.08 \pm 0.31$ \\
\hline pAHNAK-DOPC & $2.32 \pm 0.21$ & $1.99 \pm 0.30$ \\
\hline
\end{tabular}

Assuming the values of the ellipsometric angle $\psi$ to be constant and the refractive indices to be similar for each of the lipid and lipid-protein systems, ${ }^{45-46}$ the value of the ellipsometric angle $\Delta$ 
is proportional to the film thickness, as previously reported. ${ }^{25}$ For DOPS, the experimental value $\left(1.79^{\circ} \pm 0.21^{\circ}\right)$ was slightly less than the expected value, suggesting a partial penetration of pAHNAK into the DOPS monolayer (as illustrated in the schematic shown above the histogram in Figure 2). Alternatively, the conformation of pAHNAK may have changed to increase the interaction with the DOPS monolayer, resulting in this low ellipsometric angle value. For the interaction between pAHNAK and the DOPE monolayer, the experimental value $\Delta_{\mathrm{PAHNAK}-\mathrm{DOPE}}$ $\left(2.08^{\circ} \pm 0.19^{\circ}\right)$ closely agreed with the expected value $\left(2.08^{\circ} \pm 0.31^{\circ}\right)$, suggesting that pAHNAK bound to the membrane surface without insertion (as depicted in Figure 2). For DOPC, the experimental value $\Delta_{\mathrm{pAHNAK}-D O P C}$ was $2.32^{\circ} \pm 0.21^{\circ}$ indicating that the pAHNAK did not insert. It appears also to form a thicker layer at the interface, i.e. one that extends further into the subphase, but the extent of this cannot concretely established, given the error on the measurement. These data were consistent with the binding parameter analysis for the three monounsaturated phospholipids described earlier, which suggested the preferential interaction order for pAHNAK of DOPS $>$ DOPE $>$ DOPC. The depth of insertion of pAHNAK into the membrane appeared to follow the same order. 


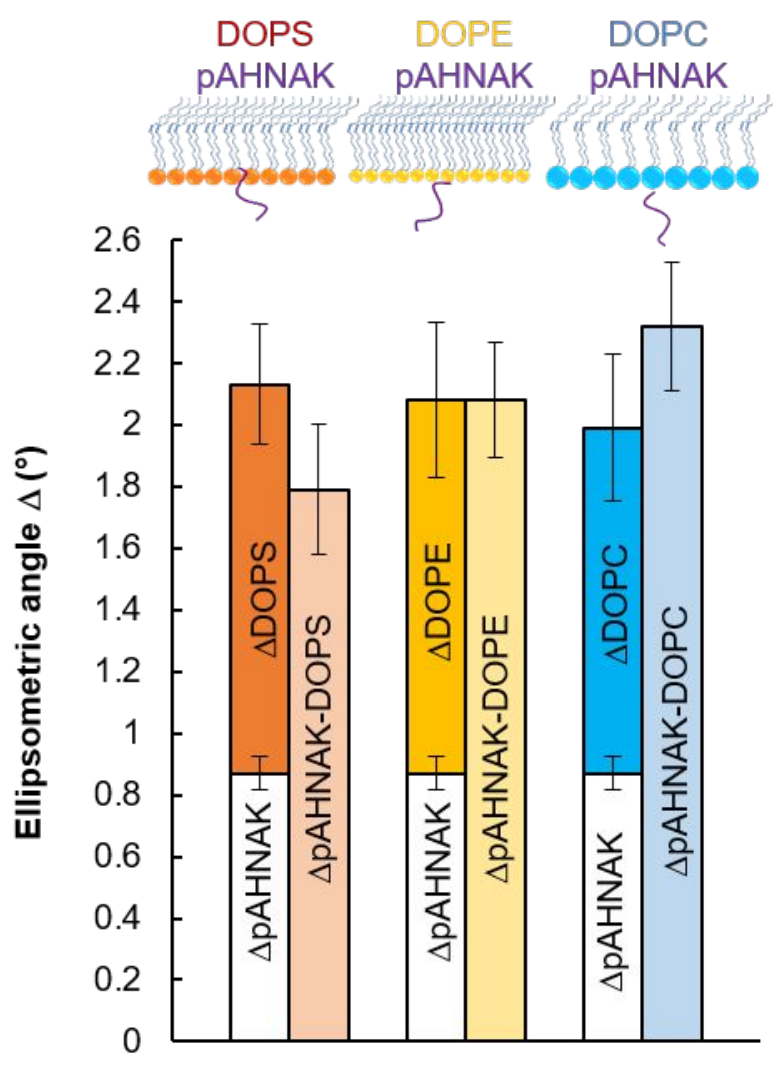

Figure 2. Expected and experimental ellipsometric angles $\Delta$ observed for pAHNAK interacting with three monounsaturated phospholipids: DOPC, DOPE, and DOPC. The illustrations above the histograms schematically show pAHNAK's interaction with each phospholipid monolayer.

Interaction Between pAHNAK and the Lipid Bilayers. The chemical-shift anisotropy (CSA) of the phosphorus atom in each phospholipid is an NMR parameter related to the inclination of the phospholipid's polar head group, the lipid phase in which it is embedded, and its order parameter. A decrease in the value of the order parameter can indicate that the phospholipid polar head group has moved toward the membrane plane or that the membrane fluidity has increased around the phospholipid. Usually, the CSA for phosphorus is determined by one-dimensional static NMR; however, the spectra for lipid mixtures overlap and CSA measurements of individual lipids in the 
mixture become difficult. Using two-dimensional NMR and magic-angle spinning, it is possible to separate the CSA of individual lipids in the fluid phase using the PROCSA pulse sequence (Figures S1 to S4). ${ }^{34}$ In the present study, the isotropic chemical shift of DOPE was set to 0 ppm, and the resonances of DOPS and DOPC appeared at -0.10 and -0.60 ppm respectively, with a precision of $\pm 0.05 \mathrm{ppm}$.

Table 3. Chemical-shift anisotropy (CSA) values of DOPE, DOPS, and DOPC at $20^{\circ} \mathrm{C}$ and $37^{\circ} \mathrm{C}$ in the absence and the presence of pAHNAK.

\begin{tabular}{|l|l|l|l|l|}
\hline Name & \multicolumn{2}{|l|}{ CSA value at $20^{\circ} \mathrm{C}(\mathrm{ppm})$} & \multicolumn{2}{l|}{ CSA value at $37^{\circ} \mathrm{C}(\mathrm{ppm})$} \\
\cline { 2 - 5 } & $\begin{array}{l}\text { Without } \\
\text { pAHNAK }\end{array}$ & $\begin{array}{l}\text { With } \\
\text { pAHNAK }\end{array}$ & $\begin{array}{l}\text { Without } \\
\text { pAHNAK }\end{array}$ & $\begin{array}{l}\text { With } \\
\text { pAHNAK }\end{array}$ \\
\hline DOPE & $21.5 \pm 0.7$ & $24.0 \pm 0.0$ & $22.5 \pm 2.1$ & $23.5 \pm 2.1$ \\
\hline DOPS & $31.5 \pm 0.7$ & $30.0 \pm 1.4$ & $31.0 \pm 0.0$ & $26.0 \pm 1.4$ \\
\hline DOPC & $26.0 \pm 0.0$ & $27.5 \pm 0.7$ & $25.5 \pm 0.7$ & $26.5 \pm 2.1$ \\
& & & & \\
\hline
\end{tabular}

Table 3 summarizes the CSA values for each phospholipid when PROCSA sequences were applied to lipid bilayers alone and in the presence of pAHNAK. The CSA results at $20^{\circ} \mathrm{C}$ and $37^{\circ} \mathrm{C}$ are shown in Figure $3 \mathrm{a}$ and $3 \mathrm{~b}$, respectively. At $20^{\circ} \mathrm{C}$, the CSA for DOPE increased slightly with pAHNAK from $21.5 \pm 0.7$ to $24.0 \pm 0.0 \mathrm{ppm}$, indicating that adding the protein resulted in the DOPE polar head group becoming more rigid. The CSA value for DOPS slightly decreased, and 
that of DOPC slightly increased, suggesting increased dynamics for DOPS and the opposite for DOPC, but those changes were within experimental error. At $37^{\circ} \mathrm{C}$, the CSA values for DOPE and DOPC did not change significantly with the addition of pAHNAK. However, the CSA value for DOPS decreased from $31.0 \pm 0.0$ to $26.0 \pm 1.4 \mathrm{ppm}$ at $37^{\circ} \mathrm{C}$ in the presence of pAHNAK, suggesting that the protein increased mobility of this polar head group region. Indeed, even if the phospholipids are already in a fluid phase, different parameters can increase the fluidity, and thus the mobility, of the lipids, such as an increasing temperature ${ }^{47-48}$ or interaction with pAHNAK in our case. Additional experiments between $5{ }^{\circ} \mathrm{C}$ and $40^{\circ} \mathrm{C}$ were performed with the three phospholipids composed of DO acyl chains and the results are detailed in Table S1. Whereas the CSA values for DOPE and DOPC remain stable, a decrease is observed for DOPS from $25^{\circ} \mathrm{C}$ and above. With this model, the interaction between pAHNAK and the polar head group of DOPS thus becomes more prominent above $25^{\circ} \mathrm{C}$. 
These findings suggest two hypotheses for each temperature. At $20^{\circ} \mathrm{C}$, pAHNAK does not insert fully into the membrane. An insertion into the DOPS film may occur, in agreement with the ellipsometry measurements, but with limited impact on the phosphorous order parameter. On the other hand, with the DOPE phospholipid head groups, the interaction results in an increase in the rigidity of the polar head groups. Given the tight hydration shell around the smaller phosphoethanolamine polar head groups,${ }^{49}$ any interaction of the pAHNAK could potentially disrupt this shell, having a more significant impact on fluidity. As with the previous experiments, a limited impact is observed with DOPC. At the physiologically relevant $37^{\circ} \mathrm{C}$, no drastic change is observed with DOPC and DOPE. However, the greater fluidity of the DOPS bilayer with the higher temperature allows the insertion of pAHNAK, resulting in the polar head groups having more room to move around and reducing their order parameter. Moreover, the structure of pAHNAK is not modified in the presence of multilamellar vesicles, as revealed by attenuated total reflection infrared (ATR) spectra (Figure S5), meaning that these changes are not due to a structure rearrangement but mainly to the insertion of the peptide into the phospholipids.

\section{SUMMARY AND CONCLUSIONS}

The Langmuir monolayer experiments showed that pAHNAK interacted more strongly with unsaturated lipids, particularly monounsaturated lipids, than with saturated lipids. The strength of the interaction between pAHNAK and the monounsaturated phospholipids found in physiological environments decreased in the order DOPS $>$ DOPE $>$ DOPC. Ellipsometry measurements of the insertion depth of pAHNAK into monolayers of these three monounsaturated lipids followed the same trend. Two-dimensional ${ }^{31} \mathrm{P}$ solid-state NMR studies of multilamellar vesicles revealed two 
different interaction modes: at $20^{\circ} \mathrm{C}$, pAHNAK significantly impacted the polar head group of DOPE, whereas at $37^{\circ} \mathrm{C}$, it was able to insert into the acyl chain of DOPS.

In conclusion, in a physiological environment at $37^{\circ} \mathrm{C}$, AHNAK can probably interact strongly with monounsaturated phospholipids with negatively charged polar head groups, with insertion into the acyl chains. This finding represents a first step in an improved understanding of AHNAK membrane behavior. Membrane repair is dependent on a large number of parameters, such as the concentration of ions, the recruitment of proteins and vesicles allowing the repair, or the composition of the membrane. ${ }^{50-51}$ When the integrity of the membrane is breached, the surrounding phospholipids will thus influence the membrane repair mechanism. Moreover, the phospholipid composition of the membrane domains can be modified or altered with some pathologies, as revealed by several lipidomic analyses. ${ }^{52-55}$ DOPS-rich domains could allow faster recruitment of AHNAK by the S100A10-annexin A2 complex, resulting in more efficient membrane repair. Future studies of the membrane interactions of other proteins in the dysferlin membrane repair complex will help to complete the map of membrane repair, potentially allowing the identification of the conditions that result in changes to these membrane bindings and possibly the loss of protein function.

\title{
AUTHOR INFORMATION
}

\section{Corresponding Author}

Email: Elodie.Boisselier@fmed.ulaval.ca

\author{
Author Contributions \\ EB, XY, IM and DEW designed the study. DEW and CED contributed to NMR and ellipsometry \\ setup, respectively. XY performed and analyzed the binding parameters and ellipsometric studies
}


of pAHNAK. FN contributed to binding parameters studies. DEW performed the ${ }^{31} \mathrm{P}$ solid-state NMR study and analyzed the data. XY and EB prepared the manuscript. DEW, IM, and CED reviewed and edited the manuscript. All the authors read and approved the final version of the manuscript.

\section{Notes}

The authors declare no competing financial interest.

\section{ACKNOWLEDGMENTS}

The authors are indebted to the Natural Sciences and Engineering Research Council of Canada (NSERC, \#RGPIN-2015-04815), the Quebec Network for Research on Protein Function, Engineering, and Applications (PROTEO), the Eye Disease Foundation and the CHU de Québec Foundation for their financial support. EB is a research scholar from the Fonds de recherche du Québec - Santé (FRQS, \#33036) in partnership with the Antoine Turmel Foundation. XY is a PhD student and FN is a MSc student, both of them received a scholarship from PROTEO. We also would like to thank Rolf Schmidt for his help in setting up ellipsometry.

\section{Supporting Information}

2D-PROCSA ${ }^{31} \mathrm{P}$ solid-state NMR spectra at $20^{\circ} \mathrm{C}$ and $37^{\circ} \mathrm{C}$ are shown in Figure S1 and S3 respectively. Extracted 1D-slices for DOPE, DOPS and DOPC (before and after adding pAHNAK) are shown in Figure S2 and S4 respectively. ATR spectra of pAHNAK alone and in the presence of multilamellar vesicles are shown in Figure S5. The influence of the temperature from $5^{\circ} \mathrm{C}$ to $40^{\circ} \mathrm{C}$ on the CSA values for DOPE, DOPS and DOPC in the presence of pAHNAK are described in Table S1. 


\section{REFERENCES}

1. Davis, T. A.; Loos, B.; Engelbrecht, A. M., AHNAK: the giant jack of all trades. Cell Signal 2014, 26 (12), 2683-93.

2. Shtivelman, E.; Cohen, F.; Bishop, J., A human gene (AHNAK) encoding an unusually large protein with a 1.2-microns polyionic rod structure. Proc Natl Acad Sci U S A. 1992, 89 (12), 5472-6.

3. Sekiya, F.; Bae, Y.; Jhon, D.; Hwang, S.; Rhee, S., AHNAK, a Protein That Binds and Activates Phospholipase C- $\gamma 1$ in the Presence of Arachidonic Acid. J Biol Chem 1999, 274 (20), $13900-7$

4. Lee, I. H.; Lim, H. J.; Yoon, S.; Seong, J. K.; Bae, D. S.; Rhee, S. G.; Bae, Y. S., Ahnak protein activates protein kinase $\mathrm{C}(\mathrm{PKC})$ through dissociation of the PKC-protein phosphatase 2A complex. J Biol Chem 2008, 283 (10), 6312-20.

5. Sussman, J.; Stokoe, D.; Ossina, N.; Shtivelman, E., Protein kinase B phosphorylates AHNAK and regulates its subcellular localization. J Cell Biol 2001, 154 (5), 1019-30.

6. Bers, D., Calcium Fluxes Involved in Control of Cardiac Myocyte Contraction. Circ Res. 2000, 87 (4), 275-81.

7. Catterall, W. A.; Perez-Reyes, E.; Snutch, T. P.; Striessnig, J., International Union of Pharmacology. XLVIII. Nomenclature and structure-function relationships of voltage-gated calcium channels. Pharmacol Rev 2005, 57 (4), 411-25.

8. Chieregatti, E.; Meldolesi, J., Regulated exocytosis: new organelles for non-secretory purposes. Nature Reviews Molecular Cell Biology 2005, 6 (2), 181-187. 
9. Borgonovo, B.; Cocucci, E.; Racchetti, G.; Podini, P.; Bachi, A.; Meldolesi, J., Regulated exocytosis: a novel, widely expressed system. Nat Cell Biol 2002, 4 (12), 955-62.

10. Cacciottolo, M.; Belcastro, V.; Laval, S.; Bushby, K.; di Bernardo, D.; Nigro, V., Reverse engineering gene network identifies new dysferlin-interacting proteins. J Biol Chem 2011, 286

(7), 5404-13.

11. de Morree, A.; Hensbergen, P. J.; van Haagen, H. H.; Dragan, I.; Deelder, A. M.; t Hoen, P. A.; Frants, R. R.; van der Maarel, S. M., Proteomic analysis of the dysferlin protein complex unveils its importance for sarcolemmal maintenance and integrity. PLoS One 2010, 5 (11), e13854.

12. Leung, C.; Utokaparch, S.; Sharma, A.; Yu, C.; Abraham, T.; Borchers, C.; Bernatchez, P., Proteomic identification of dysferlin-interacting protein complexes in human vascular endothelium. Biochem Biophys Res Commun 2011, 415 (2), 263-9.

13. Rezvanpour, A.; Santamaria-Kisiel, L.; Shaw, G. S., The S100A10-annexin A2 complex provides a novel asymmetric platform for membrane repair. J Biol Chem 2011, 286 (46), 4017483.

14. Draeger, A.; Monastyrskaya, K.; Babiychuk, E. B., Plasma membrane repair and cellular damage control: the annexin survival kit. Biochem Pharmacol 2011, 81 (6), 703-12.

15. Han, R.; Campbell, K., Dysferlin and muscle membrane repair. Curr Opin Cell Biol. 2007, 19 (4), 409-16.

16. Idone, V.; Tam, C.; Andrews, N. W., Two-way traffic on the road to plasma membrane repair. Trends Cell Biol 2008, 18 (11), 552-9. 
17. Dempsey, B. R.; Rezvanpour, A.; Lee, T. W.; Barber, K. R.; Junop, M. S.; Shaw, G. S., Structure of an asymmetric ternary protein complex provides insight for membrane interaction. Structure 2012, 20 (10), 1737-45.

18. Cocucci, E.; Racchetti, G.; Podini, P.; Meldolesi, J., Enlargeosome traffic: exocytosis triggered by various signals is followed by endocytosis, membrane shedding or both. Traffic 2007, $8(6), 742-57$.

19. Cocucci, E.; Racchetti, G.; Podini, P.; Rupnik, M.; Meldolesi, J., Enlargeosome, an exocytic vesicle resistant to nonionic detergents, undergoes endocytosis via a nonacidic route. Mol Biol Cell 2004, 15 (12), 5356-68.

20. Boisselier, E.; Demers, E.; Cantin, L.; Salesse, C., How to gather useful and valuable information from protein binding measurements using Langmuir lipid monolayers. Adv Colloid Interface Sci 2017, 243, 60-76.

21. Stefaniu, C.; Brezesinski, G.; Mohwald, H., Langmuir monolayers as models to study processes at membrane surfaces. Adv Colloid Interface Sci 2014, 208, 197-213.

22. Page, R. C.; Li, C.; Hu, J.; Gao, F. P.; Cross, T. A., Lipid bilayers: an essential environment for the understanding of membrane proteins. Magn Reson Chem 2007, 45 Suppl 1, S2-11.

23. Boisselier, E.; Calvez, P.; Demers, E.; Cantin, L.; Salesse, C., Effect of oxidation of polyunsaturated phospholipids on the binding of proteins in monolayers. Colloids Surf B Biointerfaces 2013, 109, 109-14.

24. Wilhelmy, L., Uber die Abhanigkeit der Capillaritats-Constanten des Alkols von Substanz und Gestalt des benetzten festen Korpers. Ann. Physik. Lpz. 119, 177-217. 
25. Calvez, P.; Demers, E.; Boisselier, E.; Salesse, C., Analysis of the contribution of saturated and polyunsaturated phospholipid monolayers to the binding of proteins. Langmuir 2011, 27 (4), 1373-9.

26. Calvez, P.; Schmidt, T. F.; Cantin, L.; Klinker, K.; Salesse, C., Phosphatidylserine Allows Observation of the Calcium-Myristoyl Switch of Recoverin and Its Preferential Binding. J Am Chem Soc 2016, 138 (41), 13533-13540.

27. Calvez, P.; Bussieres, S.; Eric, D.; Salesse, C., Parameters modulating the maximum insertion pressure of proteins and peptides in lipid monolayers. Biochimie 2009, 91 (6), 718-33.

28. Hoareau, E.; Belley, N.; Klinker, K.; Desbat, B.; Boisselier, E., Characterization of neurocalcin delta membrane binding by biophysical methods. Colloids Surf B Biointerfaces 2019, 174, 291-299.

29. Lemire, S.; Jeromin, A.; Boisselier, E., Membrane binding of Neuronal Calcium Sensor-1 (NCS1). Colloids Surf B Biointerfaces 2016, 139, 138-47.

30. Demers, E.; Boisselier, E.; Horchani, H.; Blaudez, D.; Calvez, P.; Cantin, L.; Belley, N.; Champagne, S.; Desbat, B.; Salesse, C., Lipid Selectivity, Orientation, and Extent of Membrane Binding of Nonacylated RP2. Biochemistry 2015, 54 (16), 2560-70.

31. Boisselier, E.; Calvez, P.; Demers, E.; Cantin, L.; Salesse, C., Influence of the physical state of phospholipid monolayers on protein binding. Langmuir 2012, 28 (25), 9680-8.

32. Mansuri, E.; Zepeda-Velazquez, L.; Schmidt, R.; Brook, M. A.; DeWolf, C. E., Surface Behavior of Boronic Acid-Terminated Silicones. Langmuir 2015, 31 (34), 9331-9.

33. Pérez-Morales, M.; Pedrosa, J. M.; Martín-Romero, M. T.; Möbius, D.; Camacho, L., Reversible Trilayer Formation at the Air-Water Interface from a Mixed Monolayer Containing a 
Cationic Lipid and an Anionic Porphyrin. The Journal of Physical Chemistry B 2004, 108 (14), 4457-4465.

34. Warschawski, D. E.; Arnold, A. A.; Marcotte, I., A New Method of Assessing Lipid Mixtures by (31)P Magic-Angle Spinning NMR. Biophys J 2018, 114 (6), 1368-1376.

35. Maggio, B.; Cumar, F.; Caputto, R., Interactions of gangliosides with phospholipids and glycosphingolipids in mixed monolayers. Biochem J. 1978, 175 (3), 1113-8.

36. Lee, A. G., How lipids affect the activities of integral membrane proteins. Biochimica et Biophysica Acta (BBA) - Biomembranes 2004, 1666 (1), 62-87.

37. Demel, R. A.; Geurts van Kessel, W. S. Z., R. F.; Roelofsen, B.; van Deenen, L. L., Relation between various phospholipase actions on human red cell membranes and the interfacial phospholipid pressure in monolayers. Biochim Biophys Acta. 1975, 406 (1), 97-107.

38. Eibl, H.; Blume, A., The influence of charge on phosphatidic acid bilayer membranes. Biochim Biophys Acta. 1979, 553 (3), 476-88.

39. Moreau, H.; Pieroni, G.; Jolivet-Reynaud, C.; Alouf, J. E.; Verger, R., A new kinetic approach for studying phospholipase $\mathrm{C}$ (Clostridium perfringens alpha toxin) activity on phospholipid monolayers. Biochemistry 1988, 27 (7), 2319-23.

40. Silvius, J. R., Role of cholesterol in lipid raft formation: lessons from lipid model systems. Biochimica et Biophysica Acta (BBA) - Biomembranes 2003, 1610 (2), 174-183.

41. Marsh, D., Lateral pressure in membranes. Biochim Biophys Acta. 1996, 1286 (3), $183-$ 223.

42. Boguslavsky, V.; Rebecchi, M.; Morris, A. J.; Jhon, D. Y.; Rhee, S. G.; McLaughlin, S., Effect of monolayer surface pressure on the activities of phosphoinositide-specific phospholipase C-beta 1, -gamma 1, and -delta 1. Biochemistry 1994, 33 (10), 3032-7. 
43. Moghaddam, B.; McNeil, S.; Zheng, Q.; Mohammed, A.; Perrie, Y., Exploring the Correlation Between Lipid Packaging in Lipoplexes and Their Transfection Efficacy.

Pharmaceutics 2011, 3, 848-864.

44. Mingotaud, A. F.; Mingotaud, É.; Patterson, L. K., Handbook of Monolayers. London, 1993; Vol. 1.

45. Lopez-Montero, I.; Lopez-Navajas, P.; Mingorance, J.; Rivas, G.; Velez, M.; Vicente, M.; Monroy, F., Intrinsic disorder of the bacterial cell division protein ZipA: coil-to-brush conformational transition. FASEB J 2013, 27 (8), 3363-75.

46. Pfrang, C.; Sebastiani, F.; Lucas, C. O.; King, M. D.; Hoare, I. D.; Chang, D.; Campbell, R. A., Ozonolysis of methyl oleate monolayers at the air-water interface: oxidation kinetics, reaction products and atmospheric implications. Phys Chem Chem Phys 2014, 16 (26), 13220-8. 47. Papahadjopoulos, D.; Jacobson, K.; Nir, S.; Isac, T., Phase transitions in phospholipid vesicles. Fluorescence polarization and permeability measurements concerning the effect of temperature and cholesterol. Biochim Biophys Acta 1973, 311 (3), 330-48.

48. Al-Ahmady, Z.; Kostarelos, K., Chemical Components for the Design of TemperatureResponsive Vesicles as Cancer Therapeutics. Chemical Reviews 2016, 116 (6), 3883-3918.

49. Rhys, N. H.; Duffy, I. B.; Sowden, C. L.; Lorenz, C. D.; McLain, S. E., On the hydration of DOPE in solution. J Chem Phys 2019, 150 (11), 115104.

50. Andrews, N. W.; Corrotte, M., Plasma membrane repair. Current Biology 2018, 28 (8), R392-R397.

51. Jimenez, A. J.; Perez, F., Plasma membrane repair: the adaptable cell life-insurance. Current Opinion in Cell Biology 2017, 47, 99-107. 
52. Koehrer, P.; Saab, S.; Berdeaux, O.; Isaico, R.; Gregoire, S.; Cabaret, S.; Bron, A. M.; Creuzot-Garcher, C. P.; Bretillon, L.; Acar, N., Erythrocyte phospholipid and polyunsaturated fatty acid composition in diabetic retinopathy. PLoS One 2014, 9 (9), e106912.

53. Wojcik-Baszko, D.; Charkiewicz, K.; Laudanski, P., Role of dyslipidemia in preeclampsia-A review of lipidomic analysis of blood, placenta, syncytiotrophoblast microvesicles and umbilical cord artery from women with preeclampsia. Prostaglandins Other Lipid Mediat 2018, 139, 19-23.

54. Li, S.; Ganguli-Indra, G.; Indra, A. K., Lipidomic analysis of epidermal lipids: a tool to predict progression of inflammatory skin disease in humans. Expert Rev Proteomics 2016, 13 (5), 451-6.

55. Fonteh, A. N.; Harrington, R. J.; Huhmer, A. F.; Biringer, R. G.; Riggins, J. N.; Harrington, M. G., Identification of Disease Markers in Human Cerebrospinal Fluid Using Lipidomic and Proteomic Methods. Disease Markers 2006, 22 (1-2). 
Table of Contents:

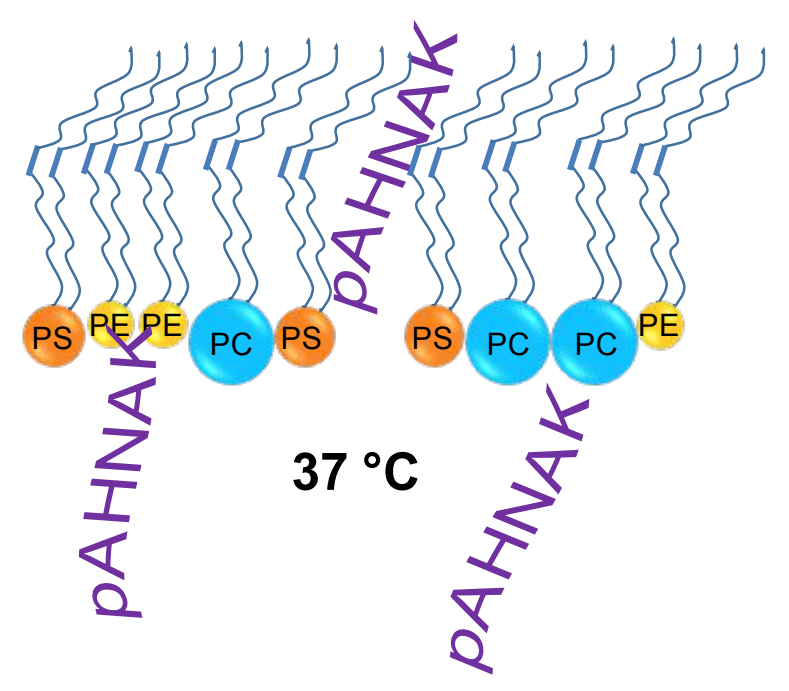

pAHNAK interacts more strongly with unsaturated lipids, particularly monounsaturated lipids, and the strength of the interaction and insertion depth decrease in the order DOPS $>$ DOPE $>$ DOPC. 